\title{
Efecto de 3 formas de fertilización en cultivo de Maíz variedad DAS 3383, La Troncal-Ecuador
}

\author{
Effect of 3 forms of fertilization on corn cultivation variety DAS 3383, \\ La Troncal-Ecuador
}

\begin{abstract}
Vera-Rodríguez JH, Cepeda-Landin WE, Cárdenas-Carreño D, Espejo-Galarza FA, Inga-Herrera GM, Balón-Cárdenas A, et al. Efecto de 3 formas de fertilización en cultivo de Maíz variedad DAS 3383, La Troncal-Ecuador. Rev Colombiana Cienc Anim. Recia. 2020; 12(1):e750. DOI: https://doi. org/10.24188/recia.v12.n1.2020.750
\end{abstract}

Universidad de Sucre, Colombia

Los autores permiten a RECIA reimprimir el material publicado en él. En caso de que un autor quiera traducir o usar una publicación parcial o completa de nuestro Diario, el autor debe obtener un permiso por escrito del editor de la revista.

Copyright (C) 2020. El (los) autor (es), Revista Colombiana de Ciencia Animal - RECIA. 2020. Este es un artículo de acceso abierto distribuido bajo los términos de Creative Commons Attribution 4.0 (https://creativecommons.org/licenses/by-nc-sa/4.0/), El uso, distribución o reproducción está permitido, siempre que se acrediten al autor original y al propietario del copyright y que se cite la publicación original en esta revista, de acuerdo con la práctica académica aceptada. No se permite el uso, distribución o reproducción que no cumpla con estos términos. 


\title{
Efecto de 3 formas de fertilización en cultivo de Maíz variedad DAS 3383, La Troncal-Ecuador
}

\author{
Effect of 3 forms of fertilization on corn cultivation variety DAS 3383, La Troncal- \\ Ecuador
}

José Humberto Vera Rodríguez. M.Sc.

Instituto Superior Tecnológico Enrique Noboa Arízaga. Carrera Tecnología en Producción Agropecuaria. La Troncal, Cañar. Ecuador. humbertorichi@hotmail.com

(io) https://orcid.org/0000-0003-3027-059X

Wilson Eder Cepeda Landin. Ing.

Instituto Superior Tecnológico Enrique Noboa Arízaga. Carrera Tecnología en Producción Agropecuaria. La Troncal, Cañar. Ecuador. ecepeda90@hotmail.com

(iD) https://orcid.org/0000-0002-3743-8378

Diana de los Ángeles Cárdenas Carreño. Tnlgo.

Instituto Superior Tecnológico Enrique Noboa Arízaga. Carrera Tecnología en Producción Agropecuaria. La Troncal, Cañar. Ecuador. dianacardenas2005@gmail.com

(iD) https://orcid.org/0000-0001-7957-0679

Felipe Abrahán Espejo Galarza. Tnlgo.

Instituto Superior Tecnológico Enrique Noboa Arízaga. Carrera Tecnología en Producción Agropecuaria. La Troncal, Cañar. Ecuador. espejofelipe97@gmail.com

(iD) https://orcid.org/0000-0002-7166-0124

Gavino Manuel Inga Herrera. Tnlgo.

Instituto Superior Tecnológico Enrique Noboa Arízaga. Carrera Tecnología en Producción Agropecuaria. La Troncal, Cañar. Ecuador gavinoingahomework@gmail.com

(id) https://orcid.org/0000-0001-9317-4736

Alexandra del Rocio Balón Cárdenas. Tnlgo.

Instituto Superior Tecnológico Enrique Noboa Arízaga. Carrera Tecnología en Producción Agropecuaria. La Troncal, Cañar. Ecuador. alexa1997@hotmail.com

iD https://orcid.org/0000-0002-2921-0752

José David Granda Correa. Tnlgo.

Instituto Superior Tecnológico Enrique Noboa Arízaga. Carrera

Tecnología en Producción Agropecuaria. La Troncal, Cañar. Ecuador. davidgranda1998@hotmail.com

(iD) https://orcid.org/0000-0001-9431-2061

Juan Carlos Delgado Orozco. Tnlgo.

Instituto Superior Tecnológico Enrique Noboa Arízaga. Carrera Tecnología en Producción Agropecuaria. La Troncal, Cañar. Ecuador. carlos.juan1995@hotmail.com

(i) https://orcid.org/0000-0001-9594-5792

\section{RESUMEN}

Se evaluó el efecto de 3 formas de fertilización vía edáfica, en maíz (Zea mays) variedad DAS 3383, sobre las variables (Altura de planta 30, 60, 90 DDS (cm); Altura de inserción de mazorca 70 DDS (cm); Longitud de mazorca (cm); Diámetro de mazorca (cm); Hileras de grano por mazorca; Peso 1000 granos (g); Relación Tusa/Grano; Rendimiento (Kg/ha ${ }^{-1}$ ); Análisis económico). Los tratamientos fueron: T1= Fertilizante enriquecido supermagro: día 8 (25\%), día 23 (25\%), día 38 (50\%); T2= Fertilizante convencional 30 g/planta: día 8 (8-20-20\% NPK), día 23 (15-3-20\% NPK), día 38 (21-0-24\% NPK); T3= Testigo (Sin fertilizante), con 4 repeticiones. El experimento se realizó en la Hacienda "Las Mercedes" del cantón La Troncal-Ecuador, duró 130 días, bajo un Diseño de Bloques Completamente Aleatorizado (DBCA), el análisis 
de datos se realizó a través del ANOVA, con la prueba de Tukey con $(\mathrm{p}<0.05)$, los cálculos se realizaron en el Paquete Estadístico InfoStaf versión 2019. Los resultados mostraron diferencias significativas a favor del tratamiento (T2) Fertilizante Convencional para las variables altura de la planta día $30 \mathrm{con} 96.40 \mathrm{~cm}$, altura inserción de la mazorca 70 días con $89.05 \mathrm{~cm}$, diámetro de la mazorca $4.76 \mathrm{~cm}$, peso de los 1000 granos 340.25 gramos y rendimiento de 9.67 Ton/ha, siendo este mismo que en el análisis económico determinó la mejor tasa de retorno marginal, dejando una tasa marginal del 9.08\% representando que por cada U\$ 1.00 invertido el productor puede recobrar su dólar invertido y obtener U\$ 0.09 adicional.

Palabras clave: Biofertilizante, fertilización, maíz, supermagro, variedad.

\begin{abstract}
The effect of 3 forms of edaphic fertilization, in corn (Zea mays) variety DAS 3383, on the variables (Plant height 30, 60, 90 DDS (cm); Cob insert height 70 DDS (cm) was evaluated; Cob length (cm); Cob diameter (cm); Rows of grain per ear; Weight 1000 grains (g); Cob / Grain ratio; Yield (Kg / ha $\left.{ }^{-1}\right)$; Economic analysis). The treatments were: T1= Supermagro enriched fertilizer: day $8(25 \%)$, day $23(25 \%)$, day 38 (50\%); T2 = Conventional fertilizer $30 \mathrm{~g} /$ plant: day 8 (8-20$20 \%$ NPK), day 23 (15-3-20\% NPK), day 38 (21-0-24\% NPK); T3 = Witness (Without fertilizer), with 4 replicas. The experiment was carried out at the farm "Las Mercedes" of the La Troncal-Ecuador canton, lasted 130 days, under a Completely Randomized Block Design (DBCA), the data analysis was carried out through ANOVA, with Tukey's test with $(\mathrm{p}<0.05)$, the calculations were performed in the InfoStaf Statistical Package version 2019. The results showed significant differences in favor of the treatment (T2) Conventional Fertilizer for the variables plant height day 30 with $96.40 \mathrm{~cm}$, height Insertion of the ear 70 days with $89.05 \mathrm{~cm}$, diameter of the cob $4.76 \mathrm{~cm}$, weight of 1000 grains 340.25 grams and yield of $9.67 \mathrm{Ton} / \mathrm{ha}$, being the same that in the economic analysis determined the best marginal return rate, leaving a marginal rate of $9.08 \%$ representing that for every U\$ 1.00 invested the producer can recover his invested dollar and obtain an additional $\mathrm{U} \$ 0.09$.
\end{abstract}

Keywords: Biofertilizer, fertilization, corn, supermagro, variety.

\title{
INTRODUCCIÓN
}

La creciente demanda de alimentos para satisfacer los requerimientos de la población y la necesidad de conservar los recursos naturales ha conducido a muchos investigadores a evaluar e implementar prácticas para aumentar la productividad de los suelos agrícolas (1). El maíz (Zea mays) es una planta originaria de América, su producto es uno de los granos básicos que alimentan a la humanidad, siendo el más importante a nivel mundial (2).

El nitrógeno es el nutriente más importante para alcanzar altos rendimientos del cultivo de maíz (3). Los productores de maíz reconocen que son necesarias, concentraciones adecuadas de fertilizante en la planta para obtener altos rendimientos, sin embargo, el dilema está en reconocer qué cantidades aplicar para lograr estas concentraciones ya que el fertilizante que no es aprovechado produce perjuicios económicos y daño ambiental (4).

Las altas dosis de fertilización química que se aplican al cultivo de maíz traen consigo una serie de problemas entre los que se destacan la contaminación de mantos freáticos, esteros y bahías; además del incremento de insectos plagas (5). El uso ineficiente de los fertilizantes, traducido como suministro excesivo de elementos químicos a los suelos de cultivo, ha sido señalado a través del tiempo, como contaminante de las áreas de producción agrícola del mundo (6).

El uso de fertilizantes orgánicos e inorgánicos se requiere en todo sistema de producción agrícola, para aumentar los rendimientos de los cultivos. La aplicación excesiva de fertilizantes inorgánicos ha generado un aumento en la contaminación del medio ambiente. Sin embargo, al aplicar fertilizantes orgánicos las condiciones del suelo mejoran, dando lugar a un aumento en el crecimiento y la producción (7). Los biofertilizantes supermagros tienen una gran importancia por su composición mineral, no está restringido, y las mejores respuestas agronómicas se observaron en las concentraciones más altas probadas, además interfieren en el control de enfermedades (8).

Por lo anterior, se planteó como objetivo evaluar el efecto de 3 formas de fertilización en cultivo de Maíz variedad DAS 3383 en el cantón La Troncal-Ecuador sobre parámetros productivos y económicos. 


\section{MATERIALES Y MÉTODOS}

Sitio de estudio. El trabajo experimental se llevó a cabo en el Recinto La Puntilla, en la Hacienda "Las Mercedes", ubicada en el Cantón La Troncal-Cañar, geográficamente ubicada en las coordenadas S2²6,18.744”, E79²3 ‘45.5424”, la misma que se encuentra en una elevación de $110 \mathrm{msnm}$, con una topografía totalmente plana, la textura del suelo es franco limoso, con un pH de 6.5, el contenido de nutrientes ug/ml (NH4 12; P 17; K 129; Ca 3165; Mg 502; S 15; Zn 3,7; Cu 10.7; Fe 212; Mn 25; B 0.30). Presenta una temperatura de media anual de $25^{\circ} \mathrm{C}$, precipitación promedio anual de $1759 \mathrm{~mm}$, heliofanía de 677 horas sol/año y velocidad del viento de $3.5 \mathrm{~m} / \mathrm{s}$ (9).

Manejo del experimento. El trabajo tuvo una duración de 130 días, utilizando como material experimental maíz de la variedad DAS 3383, en el que se evaluaron 3 formas de fertilización: T1= Fertilizante enriquecido supermagro en dosis día 8 (25\%), día 23 (25\%) y día 38 (50\%) el contenido de nutrientes de este producto fue de (N 1.2\%; P 156 ppm; K 29370 ppm); T2= Fertilizante convencional comercial 30 g/planta por aplicación en dosis día 8 (8-20-20\% NPK), día 23 (15-320\% NPK) y día 38 (21-0-24\% NPK); T3= Testigo (Sin fertilizante), para medir sus efectos sobre las variables (Altura de planta a 30, 60 y 90 DDS (cm); Altura de inserción de mazorca a los 70 DDS (cm); Longitud de mazorca (cm); Diámetro de mazorca (cm); Hileras de grano por mazorca; Peso de 1000 granos (g); Relación Tusa/Grano (\%); Rendimiento (kg/ ha-1); y Análisis económico). Las distintas formas de fertilización fueron realizadas mediante aplicación al suelo (edáfica).

Una vez iniciada la preparación del terreno, se procedió al parcelamiento del área experimental, estableciendo como unidad experimental (UE) un área de $17.50 \mathrm{~m}^{2}$ (3.50 x 5 metros), el maíz fue sembrado a una distancia de $0.80 \mathrm{~m}$ entre calles y $0.20 \mathrm{~m}$ entre plantas, dejando una semilla por hoyo perforada a punta de espeque a unos 3 a $4 \mathrm{~cm}$ de profundidad, con el fin de obtener una población de 100 plantas por UE y una población total de 12,000 plantas en estudio. Previo a la siembra se desinfectó la semilla con Thiodicarb $\left(20 \mathrm{ml} / \mathrm{Kg}^{-1}\right.$ semillas $)$, posterior a la siembra se realizó la aplicación de un herbicida pre emergente Atrazina $\left(1 \mathrm{Kg} / \mathrm{ha}^{-1}\right)+$ Paraquat $\left(1.5 \mathrm{Lts} / \mathrm{ha}^{-1}\right)$.

Se manejó el experimento bajo un Diseño de Bloques Completamente al Azar (DBCA), con 3 tratamientos y 4 repeticiones, formando un total de 12 unidades experimentales. El análisis de los datos se realizó a través del análisis de varianza (ANOVA) y para las comparaciones entre las medias se aplicó en los casos requeridos la Prueba de Tukey con (p<0.05), empleando el Paquete Estadístico InfoStaf versión 2019.

Para el análisis económico se procedió a aplicar la metodología de “Presupuestación parcial”, en la investigación de campo, al medir el efecto de 3 formas de fertilización. En este sentido, para determinar los ingresos, se ajustaron los rendimientos de campo, mediante una disminución de un 10\%, esto por asumir que el rendimiento experimental es más alto que el que obtendría el productor (10); El rendimiento ajustado, se multiplicó por el precio oficial en Ecuador de Kg de maíz (U\$ 0.34), según fuentes oficiales del Ministerio de Agricultura, Ganadería, Acuacultura y Pesca, MAGAP el 22 de abril de 2019, y se obtuvo el beneficio bruto de campo. Por la parte de costo, se determinaron aquellos costos que varían por efecto de los tratamientos para una hectárea. Al restar el total de costos que varían por el beneficio bruto de campo, se determinó el beneficio neto por tratamiento. El paso siguiente fue realizar un análisis de dominancia, que en resumen establece que "un tratamiento es dominado si presenta un beneficio neto menor a un costo mayor, que un tratamiento anterior".

Terminado el procedimiento anterior, se determinó las tasas de retorno marginal para los tratamientos no dominados, tasa que se interpreta como "el incremento porcentual en el beneficio neto debido a un incremento porcentual del $1 \%$ en los costos que varían". Por último, se realizó un análisis de sensibilidad para las tasas de retorno marginal, para establecer la relación de los costos, gastos y utilidad con respecto a las ventas (11).

\section{RESULTADOS}

Resultados Obtenidos en los Parámetros Productivos. La tabla 1, presenta los resultados obtenidos mediante el análisis de varianza para las variables estudiadas durante el tiempo de evaluación según Tukey ( $<<0.05)$ de probabilidades de error. Además, se observan sus coeficientes de variación idóneos para investigaciones de campo:

Al evaluar la altura de la planta a los 30 días, se observaron diferencias significativas ( $\mathrm{p}<0.05)$, siendo el T2 el que obtuvo una mayor altura con $96,40 \mathrm{~cm}$. Mientras que para las variables altura de la planta a los 60 y 90 días no se encontraron diferencias.

La altura de inserción de la mazorca a los 70 días presentó diferencias significativas (p<0.05), siendo el T2 el que mostró mayor promedio con $89.05 \mathrm{~cm}$. En lo que respecta la longitud de la mazorca, no se presentó diferencias significativas $(\mathrm{p}<0.05)$ al ser evaluados con los diferentes tratamientos. 
Tabla 1. Valores promedios del análisis de varianza de las variables en estudio de maíz variedad DAS 3383, bajo el efecto de 3 formas de fertilización.

\begin{tabular}{|c|c|c|c|c|c|}
\hline \multirow{2}{*}{ Variable } & \multicolumn{3}{|c|}{ Tratamientos } & \multirow{2}{*}{ E.E. } & \multirow{2}{*}{ C.V. } \\
\hline & T1 & T2 & T3 & & \\
\hline Altura de la Planta 30 días $(\mathrm{cm})$ & $92,85^{b}$ & $96,40^{\mathrm{b}}$ & $81,40^{\mathrm{a}}$ & 1,88 & 4,16 \\
\hline Altura de la Planta 60 días $(\mathrm{cm})$ & $180,50^{\mathrm{a}}$ & $261,00^{\mathrm{a}}$ & $173,00^{\mathrm{a}}$ & 35,30 & 34,47 \\
\hline Altura de la Planta 90 días $(\mathrm{cm})$ & $213,60^{\mathrm{a}}$ & $239,95^{\mathrm{a}}$ & $213,30^{\mathrm{a}}$ & 7,46 & 6,71 \\
\hline Altura inserción mazorca 70 día (cm) & $76,00^{\mathrm{a}}$ & $89,05^{\mathrm{b}}$ & $71,05^{\mathrm{a}}$ & 2,83 & 7,20 \\
\hline Diámetro de la mazorca $(\mathrm{cm})$ & $4,54^{\mathrm{a}}$ & $4,76^{\mathrm{b}}$ & $4,63^{\mathrm{ab}}$ & 0,04 & 1,85 \\
\hline Hileras de grano por mazorca & $13,80^{\mathrm{a}}$ & $13,95^{\mathrm{a}}$ & $13,70^{\mathrm{a}}$ & 0,23 & 3,32 \\
\hline Peso de 1000 Granos (g) & $312,50^{\mathrm{a}}$ & $340,25^{b}$ & $309,25^{\mathrm{a}}$ & 5,63 & 3,51 \\
\hline Relación Tuza - Grano & $5,43^{a}$ & $5,15^{\mathrm{a}}$ & $5,08^{a}$ & 0,19 & 7,38 \\
\hline Rendimiento (Ton/ha) & $8,10^{\mathrm{ab}}$ & $9,67^{b}$ & $7,89^{a}$ & 0,40 & 9,27 \\
\hline
\end{tabular}

T1 Fertilizante enriquecido supermagro; T2 Fertilización convencional; T3 Testigo (sin fertilizante).

a, b y c: letras distintas en una misma hilera indican diferencias estadísticas según Tukey $(0,05)$. EE Error estándar de la media.

$\mathbf{a}, \mathbf{b}$ and $\mathbf{c}$ : different letters in the same row indicate statistical differences according to Tukey (0.05). EE The standard error of the media.

En la variable diámetro de la mazorca, hubo diferencias significativas ( $\mathrm{p}<0.05)$, siendo el tratamiento (T2), el que presentó un mayor promedio con $4.76 \mathrm{~cm}$. En lo que respecta la variable número de hileras de grano por mazorca no presentó diferencias significativas durante el periodo de evaluación.

Al evaluar el peso de los 1000 granos, se pudo observar diferencias significativas, en la que el tratamiento T2 mostró más peso con 340.25 g. La relación tuza-grano no mostró diferencias ( $\mathrm{p}<0.05)$ al ser evaluado con los diferentes tratamientos. En cuanto al rendimiento se observaron diferencias significativas, en la que el tratamiento T2, tuvo el rendimiento máximo con 9.67 Ton/ha-1.

Análisis económico de los tratamientos en estudio en maíz variedad DAS 3383, bajo el efecto de 3 formas de fertilización. En la tabla 2, se presenta el presupuesto parcial de la investigación. Para este análisis se asumió el porcentaje del 10\% de merma de la producción para una hectárea de maíz.

Tabla 2. Presupuesto parcial de la experimentación sobre el efecto de 3 formas de fertilización en maíz variedad DAS 3383.

\begin{tabular}{|c|c|c|c|}
\hline \multirow{2}{*}{ CONCEPTO } & \multicolumn{3}{|c|}{ TRATAMIENTOS } \\
\hline & T1 & T2 & T3 \\
\hline Rendimiento medio de Maíz (Kg/ha) & 8100,00 & 9670,00 & 7890,00 \\
\hline Rendimiento Ajustado de Maíz (Kg/ha) & 7290,00 & 8703,00 & 7101,00 \\
\hline Beneficio Bruto (U\$/Kgs maíz/ha) & $2.478,60$ & $2.959,02$ & $2.414,34$ \\
\hline Preparación del Suelo (U\$/ha) & 145,00 & 145,00 & 145,00 \\
\hline Siembra (U\$/ha) & 326,90 & 326,90 & 326,90 \\
\hline Fertilizante (U\$/ha) & 242,00 & 282,00 & 0,00 \\
\hline Aplicación insecticida (U\$/ha) & 149,42 & 149,42 & 149,42 \\
\hline Cosecha (U\$/ha) & 225,00 & 225,00 & 225,00 \\
\hline Total de Costos que Varían (U\$/ha) & 1243,58 & 1283,58 & 1001,58 \\
\hline Beneficio Netos (U\$) & 1235,02 & 1675,44 & 1412,79 \\
\hline
\end{tabular}

T1 Fertilizante enriquecido supermagro; T2 Fertilización convencional; T3 Testigo (sin fertilizante)

Análisis de Dominancia. En la tabla 3, se presenta el análisis de dominancia sobre el efecto de 3 formas de fertilización en maíz variedad DAS 3383. El tratamiento (T1) Fertilizante enriquecido supermagro se le codifica con la letra D y es excluido por presentar menores beneficios netos por lo tanto se lo descarta para posteriores análisis.

Curva de Beneficios Netos. En la Figura 1, se representa la curva de beneficio neto del tratamiento (T1) Fertilizante enriquecido supermagro al tratamiento (T2) Fertilización convencional se presentó un incremento de $9.08 \%$. 
Tabla 3. Análisis de dominancia sobre el efecto de 3 formas de fertilización en maíz variedad DAS 3383.

\begin{tabular}{ccc} 
Tratamientos & Producción Kg Maíz/ha-1 & $\begin{array}{c}\text { Total de Costos (U\$) } \\
\text { que Varían }\end{array}$ \\
\hline T1 Fertilizante enriquecido supermagro & 8100,00 & 1243,58 \\
T2 Fertilización convencional & 9670,00 & 1283,58 \\
T3 Testigo (sin fertilizante) & 7890,00 & 1001,58 \\
\hline
\end{tabular}

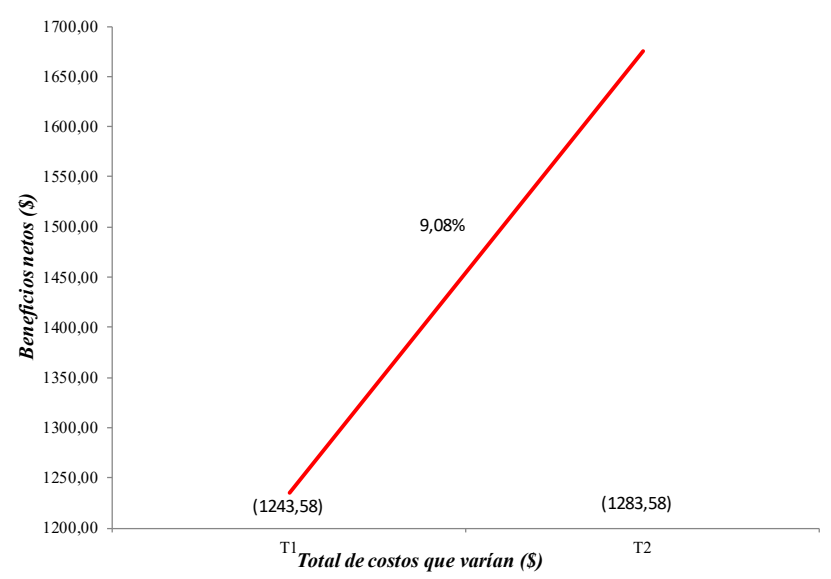

Figura 1. Curva de beneficio neto de los tratamientos (T1) Fertilizante enriquecido supermagro y tratamiento (T2) Fertilización convencional en maíz variedad DAS 3383.

Análisis Marginal. En la tabla 4, se incorpora el análisis marginal sobre el efecto de 3 formas de fertilización en maíz variedad DAS 3383. La tasa de retorno marginal indica lo que el agricultor puede esperar ganar, en promedio con su inversión cuando decide cambiar la práctica de, no adicionar fertilizante enriquecido supermagro, al decidir adicionar fertilizante convencional. Este cuadro indica que al pasar del tratamiento con fertilizante enriquecido supermagro (T1) al tratamiento con fertilización convencional (T2), representa que por cada U\$ 1.00 invertido en adicionar fertilizante convencional al maíz variedad DAS 3383, el productor puede esperar recobrar su U\$ 1.00 invertido y obtener U\$ 0.09 adicional.

Por la concerniente, el análisis económico encontró, que la mejor tasa de retorno marginal la obtuvo el tratamiento (T2) fertilización convencional con 9.08\%, logrando un beneficio neto favorable.

Tabla 4. Análisis marginal sobre el efecto de 3 formas de fertilización en maíz variedad DAS 3383.

\begin{tabular}{|c|c|c|c|c|c|}
\hline Tratamientos & $\begin{array}{c}\text { Costos que Varían } \\
\text { (U\$) }\end{array}$ & $\begin{array}{c}\text { Costos Marginales } \\
\text { (U\$) }\end{array}$ & $\begin{array}{l}\text { Beneficios Netos } \\
\text { (U\$) }\end{array}$ & $\begin{array}{c}\text { Beneficios } \\
\text { Marginales (U\$) }\end{array}$ & $\begin{array}{c}\text { Tasa de retorno } \\
\text { Marginal (\%) }\end{array}$ \\
\hline \multirow{2}{*}{$\begin{array}{l}\text { T1 Fertilizante enriquecido } \\
\text { supermagro }\end{array}$} & 1243,58 & & 1235,02 & & \\
\hline & & 40 & & 440,42 & 9,08 \\
\hline T2 Fertilización convencional & 1283,58 & & 1675,44 & & \\
\hline
\end{tabular}

\section{DISCUSIÓN}

Yanez et al (1) encontraron diferencias de altura de plantas de maíz cuando utilizaron altas dosis de fertilizante a base de nitrógeno en dosis mayores a los $80 \mathrm{~kg} / \mathrm{ha}^{-1}$. Randall et al (12) no encontraron diferencias en el desarrollo de la planta de maíz al aplicar el $\mathrm{N}$ a la siembra, o al parcializar la dosis un 30\% en la siembra y el $70 \%$ pasada la etapa V16, que corresponde a la etapa de grano lechoso. El nitrógeno es el nutriente más importante para alcanzar altos rendimientos del cultivo de maíz (3). 
La altura de inserción de la mazorca, tal vez se deba a la constitución genética propia de cada material genético influenciado por el nivel de fertilización (13). La longitud de la mazorca pudiera estar relacionado con el aporte de nitrógeno durante la fertilización (14). Mientras que Meneses et al (15) al aumentar las dosis de potasio dentro de la fertilización foliar en maíz superdulce variedad GSS 41243, no encontraron diferencias para el diámetro de la mazorca. Además, Lemcoff et al (16), indican que a mayor dosis de fertilización mayor sería el número de granos por mazorca. La aplicación de fertilizantes permite que el peso de semillas sea significativamente mayor (17).

Medina (18) manifiesta que, el propósito de una aplicación de fertilizantes es suministrar una cantidad razonable de nutrientes cuando lo demande el cultivo durante sus diferentes etapas de desarrollo y que la mayor o menor cantidad de granos y su peso es el resultado de la fotosíntesis y la respiración; estas son actividades que están influenciadas directa o indirectamente por el contenido de nutrientes y de esto dependerá la relación tuza-grano.

Reyes et al (19) observaron que incrementó el rendimiento del grano de 27.98\% (1.67 t ha $\mathrm{a}^{-1}$ ) con respecto al testigo absoluto, al utilizar biofertilizante Azospirillum brasilense, lográndo un mayor beneficio neto. El rendimiento de grano en el maíz (Zea mays L.) puede verse limitado por el suministro de carbono y/o nitrógeno a través del número reducido de granos, debido al lento crecimiento de las plantas, evitando la polinización y a través del tamaño reducido del grano debido a la disminución y/o disminución de las células de endospermo (14).

Para aumentar los ingresos del agricultor, es importante centrarse en los beneficios netos y no solamente en los rendimientos, el objetivo del análisis marginal, da la oportunidad a los tomadores de decisiones económicas, de puntualizar y discernir en qué fase se deben tomar las decisiones para re direccionar el negocio, corregir un objetivo, formular una estrategia, variar el precio del producto, entre otros (11).

En conclusión, la variedad de maíz DAS 3383 respondió favorablemente al tratamiento (T2) fertilizante convencional, registrando una mayor altura de la planta al día 30 con $96.40 \mathrm{~cm}$, posiblemente influenciado también a las propiedades nutricionales del suelo; en lo que respecta a la altura de inserción de la mazorca a los 70 días con $89.05 \mathrm{~cm}$, un diámetro de la mazorca de $4.76 \mathrm{~cm}$, en el peso de los 1000 granos alcanzó una media de 340.25 gramos y un rendimiento de 9.67 Ton/ ha $^{-1}$. Sin embargo, los distintos tipos de fertilización no incidieron sobre la altura de la planta a los 60 y 90 días, número de hileras de granos por mazorca, longitud de la mazorca y la relación tusa-grano. Mientras que, en base al análisis de presupuesto parciales, la mejor tasa de retorno marginal la obtuvo el tratamiento (T2) fertilización convencional, dejando una tasa marginal del 9.08\% que representa que por cada U\$ 1.00 invertido en fertilización convencional el productor puede recobrar su dólar invertido y obtener U\$ 0.09 adicional.

\section{Conflicto de intereses}

Declaramos no tener conflictos de interés con respecto al trabajo presentado en este informe.

\section{REFERENCIAS}

1. Yanez PB, Ríos JG, Sandoval BF, Cossio FG. Cobertura vegetativa y fertilización nitrogenada en la producción de maíz. Terra Latinoamericana. 1998; 16(3):231-237. http://www.redalyc.org/articulo.oa?id=57316306

2. Kato TA, Mapes C, Mera LM, Serratos JA, Bye RA. Origen y diversificación del maíz: una revisión analítica. Universidad Nacional Autónoma de México, Comisión Nacional para el Conocimiento y Uso de la Biodiversidad. México, DF. 2009; 116. http://www.sidalc.net/cgi-bin/wxis.exe/?IsisScript=sibe01. xis\&method $=$ post \&formato $=2 \&$ cantidad $=1 \&$ expresion $=m f n=032012$

3. Alvarez RS, Chuquija JC, Mendoza CC, Panizo RS, Sevillano RB. Fuentes y dosis de nitrógeno en la productividad del maíz amarillo duro bajo dos sistemas de siembra. In Anales Científicos. 2017; 78(2):232-240. http://dx.doi. org/10.21704/ac.v78i2.1061

4. Burkart MR, James DE. Agricultural-nitrogen contributions to hypoxia in the Gulf of Mexico. Journal of Environmental Quality. 1999; 28(3):850-859. https://dl.sciencesocieties.org/publications/jeq/abstracts/28/3/IEQ0280030850 
5. López Meza M. Efecto de la fertilización Química-Orgánica en la incidencia de Caliothrips phaseoli Hood en plantas maíz (Zea mayz L.). Revista Científica Biológico Agropecuaria Tuxpan. 2016; 6(1):166-171. http://revistabioagro. mx/wp-content/uploads/2017/01/Efecto-de-fertilización-Química-Orgánica-en-incidencia-de-Caliothripsphaseoli-Hood-en-plantas-maíz-Zea-mayz-L..pdf

6. Añez B, Espinoza W. Fertilización química y orgánica iefectos interactivos o independientes sobre la producción de zanahoria? Rev. Forest. Venez. 2002; 46(2):47-54. https://pdfs.semanticscholar. org/16b0/9524f84a0a276bea74a97b8079393cd83713.pdf

7. De Luna-Vega A, García-Sahagún ML, Rodríguez-Guzmán E, Pimienta-Barrios E. Evaluación de composta, vermicomposta y excreta de bovino en la producción de maíz (Zea mays L.). Naturales y Agropecuarias. 2016; 3(8):46-52. https://www.ecorfan.org/bolivia/researchjournals/Ciencias Naturales y Agropecuarias/vol3num8/ Revista_Ciencias Naturales V3 N8 7.pdf

8. Mazaro SM, Mangnabosco MC, Citadin I, Paulus D, de Gouvea A. Produção e qualidade de morangueiro sob diferentes concentrações de calda bordalesa, sulfocálcica e biofertilizante supermagro. Semina. Ciências Agrárias. 2013; 1(34): 3285-3294. http://dx.doi.org/10.5433/1679-0359.2013v34n6Supl1p3285

9. INAMHI. Anuario Meteorológico 2013. Instituto Nacional de Meteorología e Hidrología. Quito-Ecuador. 2013: http:// www.serviciometeorologico.gob.ec/docum institucion/anuarios/meteorologicos/Am 2013.pdf

10. Rodríguez JHV, Pinargote MVV, Cedeño JOM. Adición de carbonato cálcico y su repercusión económica sobre el grosor del cascarón en ponedoras. Revista ESPAMCIENCIA. 2012; 3(1): 1-7. http://190.15.136.171/index.php/ Revista ESPAMCIENCIA/article/view/46

11. Campos SA, Castro GH. Análisis marginal y estrategia aplicado a una cooperativa de cogestión en Costa Rica. Revista ABRA. 2019; 39(58): 65-91. https://doi.org/10.15359/abra.39-58.3

12. Randall GW, Iragavarapu TK, Bock BR. Nitrogen application methods and timing for corn after soybean in a ridge-tillage system. J Prod Agric. 1997; 10:300-307. https://dl.sciencesocieties.org/publications/ipa/abstracts/10/2/300

13. Dávila Camacho GM. Evaluación agronómica de tres híbridos de maíz (Zea mays L.) en lotes comerciales en la zona de Mata de Cacao, provincia de Los Ríos. (Tesis). (Ecuador): Universidad Católica de Santiago de Guayaquil. 2016. [citado 27 de febrero de 2020]. http://192.188.52.94/handle/3317/5404

14. Tindall HD. Vegetables in the tropics. Wesport, Corn., USA, The AVI Publishing Company. Macmillan Press Ltd., Hong Kong. 1983; 234. https://books.google.com.ec/books?id=DEFdDwAAQBAJ\&lpg=PA238\&dq=Vegetables\%20in\%20 the\%20tropics.\%20Westport\%2C\%20Corn\&hl=es\&pg=PA235\#v=onepage\&q\&f=false

15. Meneses N, Mendoza-Cortez JW, Cecílio-Filho, AB. Fertilización potásica del maíz dulce en suelo con alta disponibilidad de potasio. Agrociencia Uruguay. 2017; 21(2):54-58. http://agrocienciauruguay.uy/ojs/index.php/agrociencia/ article/view/157

16. Lemcoff J, Loomis RS. Nitrogen and density influences on silk emergence, endosperm development, and grain yield in maize (Zea mays L.). Field Crops Res. 1994; 38(2):63-72. https://doi.org/10.1016/0378-4290(94)90001-9

17. Virgen-Vargas JJ, Arellano-Vázquez I, Rojas-Martínez MA, Ávila-Perches, Gutiérrez-Hernández F. Producción de semilla de cruzas simples de híbridos de maíz en Tlaxcala, México. Rev Fitot Mex. 2010; 33(4): 107-110. http:// www.revistafitotecniamexicana.org/documentos/33-3\%20Especial\%204/19r.pdf

18. Medina P. Evaluación del comportamiento agronómico del hibrido de maiz (Zea mays L.) DK 7088. (Tesis). (Ecuador); Universidad de Guayaquil. 2010; 40. [citado 27 de febrero de 2020]. Recuperado a partir de https:// www.monografias.com/trabajos89/comportamiento-agronomico-hibrido-maiz/comportamiento-agronomicohibrido-maiz2.shtml

19. Reyes LM, Jiménez CE., Montiel MG, Galdámez JG, Cabrera JA, Aguilar FB, Padilla EG. Biofertilización y fertilización química en maíz (Zea mays L.) en Villaflores, Chiapas, México. Siembra. 2018; 5(1): 026-037. http://200.12.169.32/ index.php/SIEMBRA/article/view/1425 